Historic, Archive Document

Do not assume content reflects current scientific knowledge, policies, or practices. 



\section{SEED LIST}

OF

\section{Native Trees, Shrubs and Plants COLLECTED BY}

L. E. WILLIAMS NURSERY CO. EXETER, N. H.

U. S. A.

The following list of Seeds we offer subject to the condition of the crop.

We collect only as ordered, and carry over no old seed. Orders should be placed before it is too late to collect them.

$11 \mathrm{~b}$.

Acer Negundo

" Pennsylvanicum ................. 1.50

" Platanoides..................... 1.00

" rubrum ......................... 1.00

" saccharum ................... 1.00

" saccharinum ................. 1.00

" spicatum .................... 1.50

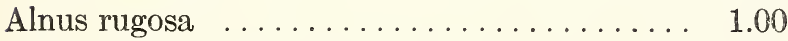

Amelanchier Canadensis, berries . . . . . . . . . 2.00

" Botryapium, berries
2.00
$10 \mathrm{lbs}$. $\$ 6.00$

7.50

9.00

7.50

7.50

7.50

15.00

15.00 
$1 \mathrm{lb}$.

Ampelopsis quinquefolia, berries ......... \$1.00

Andromeda calyculata ............... 5.00

" ligustrina .............. 5.00

Berberis thunbergi, berries . . . . . . . . . . . 1.50

" vulgaris, berries............. 1.00

Betula lenta ................... 1.25

" lutea .................... 1.25

" papyrifera ................... 1.50

" populifolia ................... 1.00

Carpinus Americana ................. 1.00

Celastrus scandens, berries ............ 1.00

Cephalanthus occidentalis . . . . . . . . . . 1.00

Clematis Virginiana ................ 3.00

Clethra alnifolia .................. 1.50

Cornus alternifolia, berries . . . . . . . . . 1.00

" Canadensis, berries ............. 1.00

" circinata, berries ............. 1.00

" Florida, berries ................ 1.00

" paniculata, berries ............. 1.00

" sericea, berries .............. 1.00

" stolonifera, berries ............. 1.00

Crataegus coccinea, berries ............ 1.00

" crusgalli, berries ............ 1.00

Diervilla trifida .................... 1.50

Dirca palustris ................... 1.50

Fraxinus Americana .................. 1.00

aultheria procumbens, berries
$10 \mathrm{lbs}$.

$\$ 7.50$

10.00

5.00

10.00

10.00

12.50

7.50

7.50

7.50

7.50

25.00

10.00

7.50

9.00

9.00

5.00

6.00

6.00

7.50

6.00

6.00

6.00

10.00 
$1 \mathrm{lb}$.

$10 \mathrm{lbs}$.

Gaylussacia frondosa, berries $\ldots . \ldots \ldots \ldots \$ 2.00$

“ risinosa, berries .......... 1.50

Gentiana Andrewsii ............... 3.00 per oz.

" crinita................ 5.00 per oz.

Hamamelis Virginica, clean seed .......... 3.00

Hicoria ovata ...................... .25

$\$ 1.50$

Ilex verticillata, berries .............. 1.00

6.00

Ilicioides mucronata, berries. . . . . . . . . . 1.00

Juniperus communis, berries ........... 1.00

6.00

" depressa, berries ........... 1.00

6.00

" Virginiana, berries ............ 2.00

15.00

Lobelia cardinalis

25.00

Myrica Carolinensis, berries ............ 1.00

6.00

Myrica cerifera, berries .............. 1.00

6.00

Ostrya Vriginica .................... 1.50

10.00

Pinus resinosa . . . . . . . . . . . . . . . . . 20.00

" rigida ................... 6.00

" strobus .................... 4.00

Prunus maritima, berries ............ 1.50

“ Pennsylvanicum, berries ......... $1.00 \quad 7.50$

" serotina, berries.............. $1.00 \quad 6.00$

"Virginiana, berries ............. $1.00 \quad 6.00$

Pyrus arbutifolia (red), berries .......... $1.00 \quad 6.00$

" nigra, berries .................. $1.00 \quad 6.00$

Rhus glabra, berries ............... $1.00 \quad 6.00$

" glabra, berries .................. $1.00 \quad 5.00$

" typhina, berries ................ $1.00 \quad 5.00$ 
$1 \mathrm{lb}$.

Rosa Carolina, berries

" humilis, berries

" lucida, berries

" nitida, berries

" rubignosa, berries

Sambucus Canadensis, berries

Smilax herbatia, berries

" rotundifolia, berries

Sorbus Americana, berries

Spiraea salicifolia

" tomentosa

Tilla Americana, berries

Tsuga Canadensis,

Vaccinium atrococcum, berries

Vaccinium corymbosum, berries

66

66

macrocarpon, berries

nigrum, berries

palladium, berries

Pennsylvanicum, berries

vacillans, berries

Viburnum acerfolium, berries

6

66

cassinoides, berries

dentatum, berries

lantanoides, berries

Lentago, berries

Americana, berries
$\$ 1.00$

1.00

1.00

1.00

1.00

1.00

1.00

1.00

1.00

1.00

1.00

1.00

6.00

1.00

1.00

.50

1.00

1.00

1.00

1.00

1.00

1.00

1.00

1.60

1.00

1.00
10 lbs.

$\$ 5.00$

6.00

6.00

6.00

6.00

6.00

6.00

7.50

5.00

5.00

7.50

50.00

9.00

9.00

2.50

9.00

9.00

9.00

9.00

6.00

6.00

6.00

6.00

6.00

6.00 